\title{
An Evaluation of Open Source E-Learning Platforms Stressing Adaptation Issues*
}

\author{
Sabine Graf and Beate List \\ Women's Postgraduate College of Internet Technologies \\ Vienna University of Technology \\ \{graf, list\}@wit.tuwien.ac.at
}

\begin{abstract}
This paper presents an evaluation of open source $e$ learning platforms. The main focus is on adaptation issues. The result of the evaluation shows that the platform Moodle outperforms all other platforms and also obtained the best rating in the adaptation category.
\end{abstract}

\section{Introduction}

Adaptation received very little coverage in e-learning platforms. An e-learning course should not be designed in a vacuum; rather, it should match students' needs and desires as closely as possible, and adapt during course progression. This paper presents an evaluation of open source e-learning platforms with the aim of finding the platform most suitable for extending to an adaptive one. The extended platform will be utilized in an operational teaching environment. Therefore, the overall functionality of the platform is as important as the adaptation capabilities, and the evaluation treats both issues.

To our knowledge, there are only a few e-learning platform evaluations available in the current literature. Their main focus is on commercial products. In contrast, this work is focused on open source products only. In [3] and [10] general purpose evaluations have been conducted. Both applied a simple evaluation approach. In contrast, the evaluation described in [2], used the comprehensive qualitative weight and sum approach [14].

Our evaluation is also based on the qualitative weight and sum approach, which is described in Section 2. After a pre-evaluation phase, nine platforms were analyzed in detail. The detailed evaluation approach is described in Section 3 and Section 4 is focused on the adaptation category and its results. The overall evaluation results are presented in Section 5 and Section 6 concludes the paper.

\footnotetext{
* This research has been funded by the Austrian Federal Ministry for Education, Science, and Culture, and the European Social Fund (ESF) under grant 31.963/46-VII/9/2002.
}

\section{Qualitative Weight and Sum Approach}

The qualitative weight and sum (QWS) [14] approach is a well-established approach for the evaluation of software products. It establishes and weights a list of criteria. QWS is based on the use of symbols. There are six qualitative levels of importance for the weights, frequently symbols are used: $\mathrm{E}=$ essential, $*=$ extremely valuable, $\#=$ very valuable, $+=$ valuable, $\mid=$ marginally valuable and $0=$ not valuable. The weight of a criterion determines the range of values that can be used to measure a product's performance. For a criterion weighted \#, for example, the product can only be judged $\#,+, \mid$, or 0 , but not *. This means that lower-weighted criteria cannot overpower higher-weighted criteria.

To evaluate the results, the different symbols given to each product are counted. Example results can be $2 *, 3 \#$, 3 or $1^{*}, 6 \#, 1+$. The product can now be ranked according to these numbers. But the results are sometimes not clear. There is no doubt that $3^{*}, 4 \#, 2 \mid$ is better than $2^{*}, 4 \#, 2 \mid$ but it is not clear whether it is better than $2^{*}, 6 \#, 1+$. In the latter case further analysis has to be conducted.

\section{Applied Evaluation Approach}

We selected the QWS approach for this evaluation, because of the differentiated results, which highlight the strengths and limitations of the platforms. We adapted the approach in a way where the essential criteria are assessed in a pre-evaluation phase, similar to Baumgartner [2]. These minimum criteria cover three general usage requirements: an active community, a stable development status, and a good documentation of the platform. The fourth criterion incorporates the didactical objective and means that the platform's focus is on the presentation of content instead of communication functionalities.

At the beginning of the evaluation, we selected 36 platforms and evaluated these according to the minimum 
criteria. Nine platforms (ATutor 1.4.1 ${ }^{1}$ [1], Dokeos 1.5.5 [4], dotLRN 2.0.3 [5], based on OpenACS 5.1.0 [11], ILIAS 3.2.4 [7], LON-CAPA 1.1.3 [8], Moodle 1.4.1 [9], OpenUSS 1.4 [12] extended with Freestyle Learning 3.2 [6], Sakai 1.0 [13], and Spaghettilearning 1.1 [15]) meet the criteria. Next, these nine platforms were tested in detail. A questionnaire and an example of a real life teaching situation, covering instructions for creating courses, managing users and simulating course activities, were designed and applied to each platform.

Finally, we established eight categories: communication tools, learning objects, management of user data, usability, adaptation, technical aspects, administration, and course management. These categories act merely as a classification and include several subcategories. Only the subcategories are weighted and evaluated. Several attributes measure the characteristics of each subcategory. Furthermore, a rule is defined for each subcategory, which assigns the combination of measured attribute values to an evaluation value of the subcategory. According to the QWS approach, these values are summarized for each category by building the number of each symbol. The evaluation value of the platform is calculated equivalently.

\section{Adaptation Capabilities}

This section is focused on adaptability, personalization, extensibility, and adaptivity capabilities of the platforms. We focused on customizable adaptation only, which can be done without programming skills.

Adaptability includes all facilities to customize the platform for the educational institution's needs (e.g. the language or the design). Personalization aspects indicate the facilities of each individual user to customize his/her own view of the platform. Extensibility is, in principle, possible for all open source products. Nevertheless, there can be big differences. For example, a good programming style or the availability of a documented application programming interfaces (API) are helpful. Adaptivity indicates all kinds of automatic adaptation to the individual user's needs (e.g. personal annotations of learning objects or automatically adapted content).

The evaluation results of the adaptation category are presented in Table 1. The maximum values represent the values, which can be achieved at maximum per subcategory. Examining the results from a vertical perspective, it can be seen that the adaptability and the personalization subcategories yield a broad range of results. The majority of the platforms were estimated as very good with regard to extensibility. In contrast, adaptivity features are underdeveloped. The majority of platforms does not consider adaptivity at all.

\footnotetext{
${ }^{1}$ The decimal numbers refer to the version evaluated.
}

Table 1: Results of the Adaptation Category

\begin{tabular}{|c|c|c|c|c|c|}
\hline & 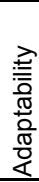 & 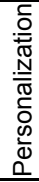 & 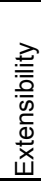 & 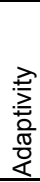 & 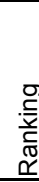 \\
\hline Maximum values & * & \# & * & * & \\
\hline ATutor & 1 & \# & $\#$ & 1 & 3 \\
\hline Dokeos & 1 & 0 & * & + & 2 \\
\hline dotLRN & + & + & * & 0 & 2 \\
\hline ILIAS & + & \# & * & 0 & 2 \\
\hline LON-CAPA & + & \# & \# & | & 2 \\
\hline Moodle & \# & + & * & | & 1 \\
\hline OpenUSS & \# & \# & \# & 0 & 2 \\
\hline Sakai & 0 & 0 & * & 0 & 3 \\
\hline Spaghettilearning & + & \# & + & 0 & 3 \\
\hline
\end{tabular}

Looking at the results in a platform specific way, it can be seen that an exact ranking is not possible. Due to the use of the QWS approach, a pairwise comparison of all platforms is necessary to determine the ranking. Because these comparisons do not result in a sequential order, the platforms need to be grouped into clusters. As a result, Moodle can be seen as the best platform concerning adaptation issues. Moodle provides an adaptive feature called "lesson" where learners can be routed automatically through pages depending on the answer to a question after each page. Furthermore, the extensibility is supported very well by a documented API, detailed guidelines, and templates for programming. Also adaptability and personalization aspects are included in Moodle. Templates for themes are available and can be selected by the administrator. Students can choose out of more than 40 languages.

\section{Evaluation Results}

This section presents the overall evaluation results. Table 2 shows the results for each platform and each subcategory, classified by categories. The best results of each category are highlighted. Moodle dominates the evaluation by achieving the best value five times. The strengths of Moodle are the realization of communication tools, and the creation and administration of learning objects. Additional strengths of Moodle are the comprehensive didactical concepts and also the tracking of data. Furthermore, the outstanding usability of Moodle leads to the maximum evaluation value in the usability category. Concerning the other platforms, ILIAS obtained the best values in the categories technical aspects, administration, and course management.

To get the overall evaluation result, the symbols of all categories need to be summarized. Figure 1 shows the results of the platforms in a descending order. Similar to the adaptation category, it is not possible to assign an 


\section{Table 2: Evaluation Results of E-Learning Platforms for each Subcategory}

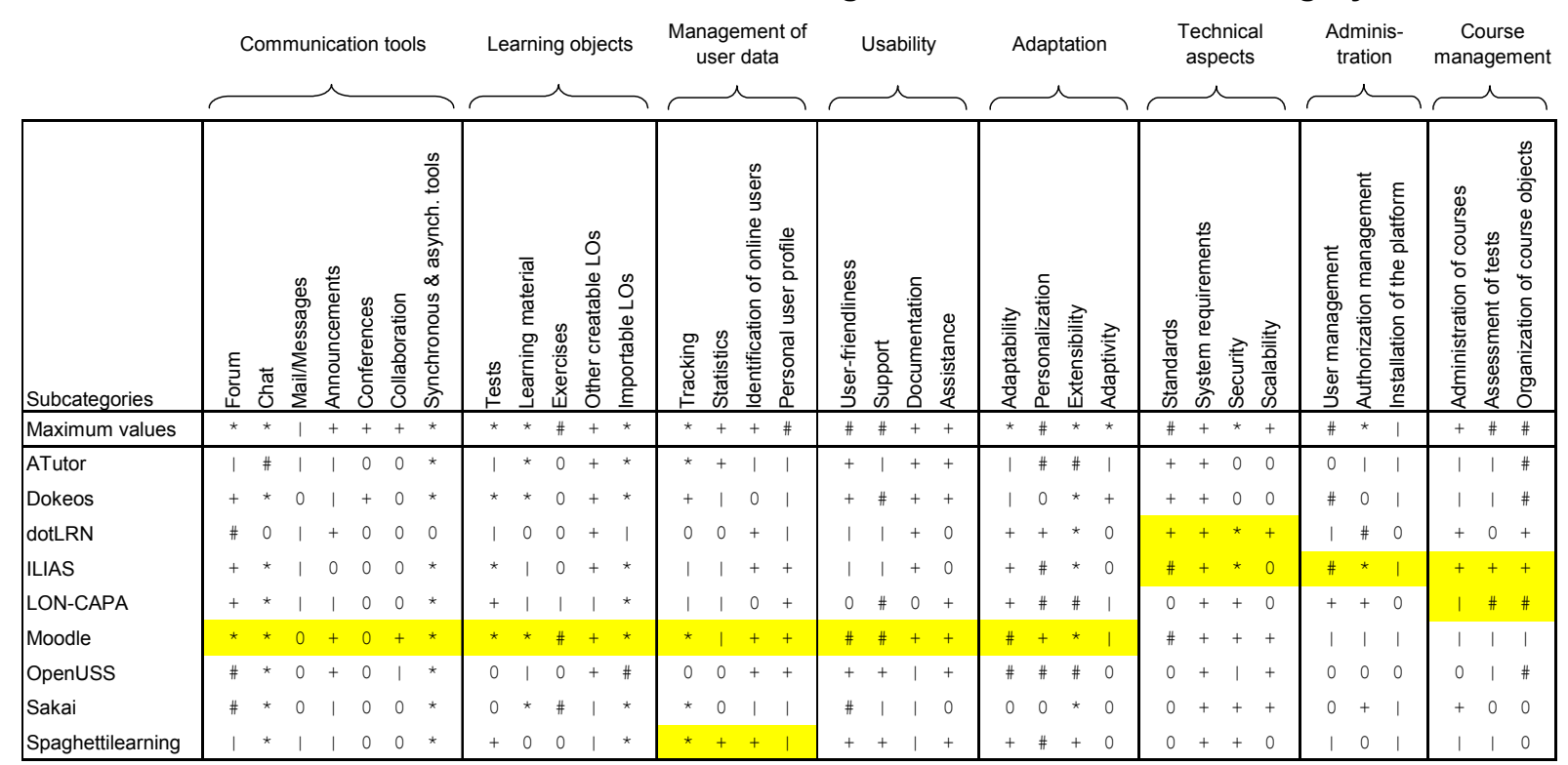

exact ranking for each platform. However, it can be seen that Moodle achieved the best evaluation values. Also the second and third rank can be assigned clearly to ILIAS and Dokeos. According to the pairwise comparisons ATutor, LON-CAPA, Spaghettilearning, and Open-USS are ranked equally at the fourth position, whereas Sakai and dotLRN are ranked last. The reason for the low ranking of Sakai is that so far only the basic features are realized. But, the quality of these features is very good.

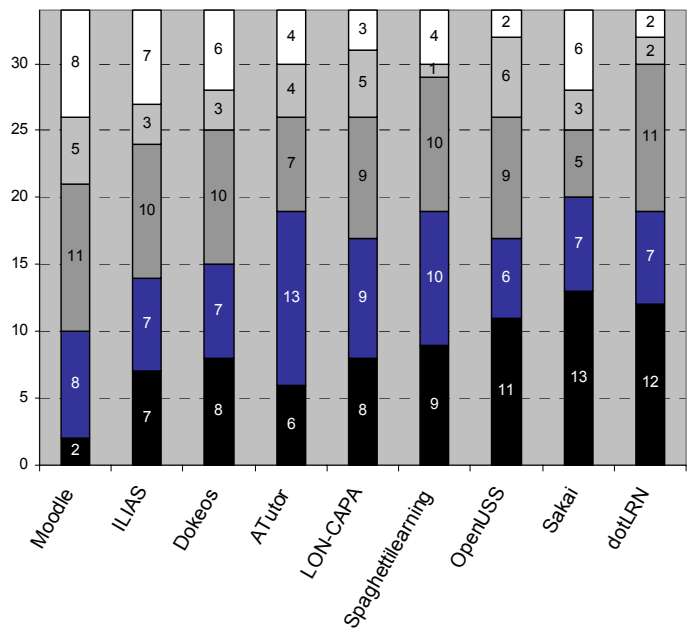

- not available / very poor (0) $\square$ poor (l) $\square$ moderate $(+)$ ugood (\#) $\square$ very good $\left(^{*}\right)$

Figure 1: Overall Evaluation Results

\section{Conclusion}

The aim of this evaluation was to identify the most suitable open source e-learning platform for extending to an adaptive one. The evaluation applies an extended qualitative weight and sum approach. After a preevaluation phase, nine platforms were analysed in detail. Moodle obtained the best results in the general as well as in the specific adaptation evaluation.

In our future work, we will extend the selected platform in a way that the courses adapt to the unique strengths, learning objectives, knowledge levels, and learning styles of each individual learner.

\section{References}

[1] ATutor, http://www.atutor.ca

[2] P. Baumgartner, H. Häfele, and K. Maier-Häfele, ELearning Praxishandbuch - Auswahl von Lernplattformen. Innsbruck: Studienverlag, 2002.

[3] F. Colace, M. DeSanto, and M. Vento, "Evaluating On-line Learning Platforms: a Case Study", in Proc. $36^{\text {th }}$ Hawaii International Conference on System Sciences, Hawaii, IEEE Press, 2003.

[4] Dokeos, http://www.dokeos.com

[5] dotLRN, http://dotlrn.org

[6] Freestyle Learning, http://www.freestyle-learning.de

[7] ILIAS, http://www.ilias.uni-koeln.de

[8] LON-CAPA, http://www.lon-capa.org

[9] Moodle, http://moodle.org

[10] M. O’Droma, I. Ganchev, and F. McDonnell, "Architectural and functional design and evaluation of e-learning VUIS based on the proposed IEEE LTSA reference model", The Internet and Higher Education, vol. 6, no. 3, Elsevier Inc., pp. 263-276, July 2003

[11] OpenACS, http://openacs.org

[12] OpenUSS, http://openuss.sourceforge.net/openuss

[13] Sakai, http://www.sakaiproject.org

[14] M. Scriven, Evaluation Thesaurus (4th ed.), Newbury Park, CA: Sage Publications, 1991.

[15] Spaghettilearning, http://www.spaghettilearning.com 